\title{
Falls and Fall Prevention
}

About a third of older persons fall at least once a year, but less than $5 \%$ of these falls result in a fracture. Wrist fractures usually involve a fall onto the outstretched arm. Proximal humerus fractures occur following a fall on the adducted arm. Hip fractures and fractures of the pelvic ring occur after a fall on the side or the back. Backwards falls with axial compression of the spine can lead to vertebral fractures.

Falling leads to increased fear of falling, resulting in reduced physical activity due to avoidance strategies. Over time immobility is also associated with increased risk of falling.

Factors associated with increased risk of falling in the frail elderly are:

- Reduced muscle mass and strength

- Slowing of reflexes

- Use of walking aids

- Loss of equilibrium

- Cognitive deficits

- Impaired vision

- Impaired coordination

- Hypnotics, sedatives and psychotropics

- Alcohol

- Environmental hazards (e.g. cables)

Studies in geriatric patients showed that $75 \%$ of patients were mobile without walking aids before the fracture but only $20 \%$ of them after the fracture. Therefore, falling represents a negative "life event" in this frail patient group. Falling can be a sign of frailty, aggravation of chronic diseases and imminent loss of independence. In frail elderly patients, presence of multiple risk factors like depression, sarcopenia, weight loss and immobility further increases the risk of falling. For identification of patients at risk and also to evaluate physical function following rehabilitation after a fragility fracture, geriatric assessment scores (e.g. Charlson co-morbidity index) and fall evaluation forms should be used to assess also the psychological, cognitive and nutrition status of the patient.

Therapeutic interventions include balance programmes, strengthening exercises, reduction of unnecessary medications, optimising lifestyle and the use of walking aids and protection devices. The Cochrane Database showed that group- and home-based exercise programmes and home safety interventions reduce the rate of falls in older adults. Vitamin D supplementation in individuals with vitamin D deficiency should be given with an initial boosting dose and consequent application of 10002000 IU daily. In several studies vitamin D supplementation showed advantages for muscle function and reduction of falls and fracture events in elderly patients with vitamin D deficiency. 